\title{
CABILDEO MEDIANTE LAS ACTIVIDADES DE RESPONSABILIDAD SOCIAL: CASO COOPERATIVA LA CRUZ AZUL
}

Recepción: 17 de Octubre del 2017; Aceptación: 17 de Novimebre del 2017

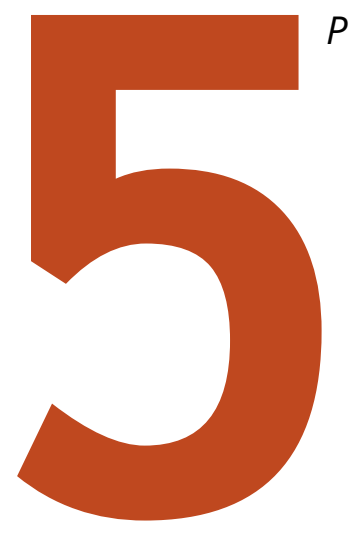

Alma Isela Rodríguez Hernández ${ }^{1}$

almis_is@hotmail.com

o 2113065224@cua.uam.mx

Universidad Autónoma Metropolitana Unidad Cuajimalpa

\section{RESUMEN}

El objetivo de este proyecto es describir la relación existente entre las Actividades de Responsabilidad Social y el cabildeo con base en la teoría de los stakeholders y las relaciones públicas. La metodología de esta investigación fue empírica, basada en la observación no participante y en entrevistas a profundidad. Se realizó una entrevista a un miembro de la Cooperativa La Cruz Azul, S. C. L., en su planta en Oaxaca. Además, se hizo un análisis teórico de los estudios previos a este proyecto.

Como resultado se sostiene la hipótesis de que al utilizar las Actividades de Responsabilidad Social en el proceso de cabildeo desarrollado por el área de relaciones públicas se puede influir en los procesos de diseño

normativo que le afectan a la empresa.
Esta investigación permite concluir que las Actividades de Responsabilidad Social y cabildeo son importantes para el desarrollo de las empresas, por ello la relevancia de integrar el tema al ámbito administrativo.

Palabras clave: Actividades de Responsabilidad Social (ARS), cabildeo, relaciones públicas de las empresas, estrategias de comunicación y pensamiento estratégico.

\section{ABSTRACT}

The objective of this project is to describe the relationship between Social Responsibility Activities and lobbying based on stakeholder theory and public relations. The methodology of this research was empirical, based on non-participant observation and in-depth interviews. An interview was made with a member of the La Cruz Azul Cooperative, S. C. L., at his plant in Oaxaca. In addition, a theoretical analysis of the studies prior to this project was made.

As a result, the hypothesis is that using the Social Responsibility Activities in the lobbying process developed by the public relations area can influence the normative design processes that affect the company. This research allows to conclude that the Social Responsibility and Lobbying activities are important for the development of the companies, for that reason the relevance of integrating the subject to the administrative scope.

Keywords: Social Responsibility Activities (ARS), lobbying, public relations of companies, communication strategies and strategic thinking. 


\section{PLANTEAMIENTO DEL PROBLEMA}

Las organizaciones económicas han incrementado sus actividades de responsabilidad social. En algunos casos dichas actividades están relacionadas directamente con el impacto causado por las operaciones de las empresas, independientemente si es responsabilidad legal o social de estas últimas.

En otros casos, hay empresas que realizan actos considerados de responsabilidad social como las campañas de reforestación, que si bien no son su obligación, dichas actividades logran integrar a diversos sectores de la sociedad. Al mismo tiempo que llegan a formar parte importante para que las empresas sean reconocidas como socialmente responsables.

Una de las estrategias de las empresas para comunicar sus Actividades de Responsabilidad Social (ARS) ha sido el surgimiento de los distintivos o procesos de certificación desarrollados por grupos empresariales. Dichas estrategias son para comunicar que las organizaciones tienen un compromiso social y que no sólo están atendiendo el cumplimiento legal, sino que van más allá. Entre ellos están la certificación de Empresa Socialmente Responsable (ESR) y el ISO 26'000.

El distintivo de ESR se genera a partir de una evaluación que realizan dos organismos formados también por organizaciones económicas, el Centro Mexicano para la Filantropía (Cemefi) y la Alianza por la Responsabilidad Social (AliaRSE), conforme a un decálogo de normas que estas mismas estipulan, con el fin de reconocer a las mejores Empresas Socialmente Responsables. Estas empresas se registran voluntariamente para participar en procesos de evaluación.

Otro tipo de distintivo es la norma ISO 26'000, la guía de responsabilidad social. Ésta contiene puntos específicos acordados por 163 países sobre los aspectos fundamentales que debe cumplir una organización, es decir, qué y cómo debe ser de manera que aspire a ser reconocida como responsable ante la sociedad. No hay requisitos legales para que las empresas se registren bajo la norma y de igual forma es voluntario.
Ha de reconocerse un incremento de certificados que acreditan las ARS de las empresas. En 2014 se dio este distintivo a 935 empresas mexicanas y para el presente año aumentó el número a 1164, con la última actualización a marzo de 2015, según datos de la Cemefi (2015).

Además, hay que resaltar que las ARS aparecen cada vez más en los medios de comunicación, formando así parte de un proceso planificado de las estrategias de relaciones públicas de las empresas.

El término relaciones públicas no es nuevo, surgió a finales del siglo XX y actualmente toma importancia por el hecho de que las empresas quieren dar a conocer sus ARS para obtener legitimidad. Es por esto que la comunicación es una herramienta para hacer llegar a un número más grande de personas la información sobre sus ARS.

Entre las estrategias de relaciones públicas podemos encontrar las actividades de comunicación y cabildeo. En la actualidad hay una incorporación de estas dos actividades a las estrategias de difusión de información de las empresas, como una forma de adquirir la legitimidad.

Es allí en la legitimación que se buscan nuevas formas de influencia sobre las personas interesadas en tener una relación con las empresas, mejor conocidas como stakeholders (Schwartz y Carroll, 2003). Cuando la legitimación es positiva, se crea un contexto legal y social beneficioso para las empresas.

Actualmente, se presentan debates públicos que cuestionan las ARS de ciertos grupos de empresas que pueden ser afectados de forma negativa. Por lo que las empresas hacen uso de estrategias de relaciones públicas, con el fin de orientar a su favor la opinión de los implicados en el debate. Esto conlleva a que las empresas utilicen estrategias para lograr una coordinación entre el discurso y la acción para convencer a las personas. 
Estos debates se realizan tanto en círculos académicos, políticos y sociales, muchos generados por los cambios institucionales del país. Por ello, las estrategias de relaciones públicas buscan siempre que la empresa esté presente en los debates que forman parte de la plataforma institucional, en la cual opera esta misma. El objetivo de esta estrategia es influir en el andamiaje institucional, desde la propuesta de la ley hasta la aplicación de las normas. Principalmente en las normas por ser las que afectan de forma más directa las actividades específicas de las empresas.

Así pues, el presente trabajo propone considerar las ARS como una herramienta útil en el proceso de cabildeo porque dota a las empresas de legitimidad para participar eficientemente en la definición de las normas que se le apliquen, basándose en la teoría de las relaciones públicas y el pensamiento estratégico.

\section{OBJETIVO GENERAL}

Describir las relaciones entre las Actividades de Responsabilidad Social y el cabildeo o lobbying, que están presentes en las estrategias de comunicación de las empresas, a partir de las teorías del pensamiento estratégico y las relaciones públicas, con el fin de mostrar su relevancia en el ámbito administrativo.

\section{OBJETIVO ESPECÍFICO}

Describir la relación que existe entre las ARS de la Cooperativa La Cruz Azul, S. C. L., y el proceso de cabildeo o lobbying, con base en sus relaciones públicas. Con el fin de corroborar la importancia que tiene la relación entre estos dos términos dentro del ámbito administrativo.

\section{MARCO TEÓRICO}

Responsabilidad Social como herramienta comunicativa en el proceso de cabildeo.

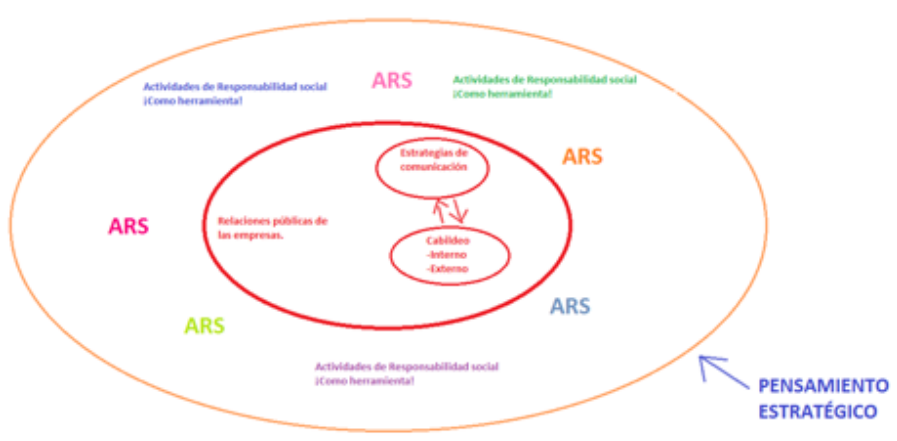

Fuente: elaboración propia.

La gráfica 1 es el modelo que representa la estructura de esta investigación y explica lo siguiente: como eje principal está la Responsabilidad Social Empresarial que tiene un sinfín de significados, sin embargo, en este caso se hace uso de la definición hecha por la Comisión Económica para América Latina y el Caribe, que se refiere a ésta como: "una forma de hacer negocios que toma en cuenta los efectos sociales, ambientales y económicos de la acción empresarial, integrando en ella el respeto por los valores éticos, las personas, las comunidades y el medio ambiente" (Correa et al., 2004). La Responsabilidad social será la herramienta que utiliza el área de las relaciones públicas para realizar sus procesos de cabildeo.

Dentro de las organizaciones, no en todas, hay un área de relaciones públicas, cuyo fin es informar sobre personas, empresas, instituciones, etc., mediante gestiones personales o con el empleo de las técnicas de difusión y comunicación, tratando de prestigiarlas y de captar voluntades a su favor (RAE, 2015).

En esta área de relaciones públicas se realizan comúnmente dos actividades, que son las estrategias de comunicación y el cabildeo. Las primeras son un proceso planificado de cómo se va a transmitir un mensaje de forma que el receptor codifique correctamente lo que se le quiere decir. 
Mientras que el cabildeo (lobbying en inglés) es "el proceso planificado de comunicación de contenido predominantemente informativo, en el marco de la política de relaciones públicas, de la empresa u organización con los poderes públicos, ejercido directamente por ésta, o a través de un tercero mediante contraprestación, que tiene como función intervenir sobre una decisión pública (norma o acto jurídico; en proyecto o en aplicación) o promover una nueva, transmitiendo una imagen positiva basada en la credibilidad de los argumentos defendidos que genere un entorno normativo y social favorable, y con la finalidad de orientarla en el sentido deseado y favorable a los intereses representados" (Xifra, 1998).

Ambos términos son muy parecidos, es por esto que resulta relevante aclarar las diferencias que tienen.

En este sentido, se considera un eje transversal, el pensamiento estratégico. "Pensar estratégicamente implica un proceso de visualizar el futuro, sembrarle objetivos y tender los puentes desde el presente considerando los cambios continuos del entorno" (Corona, 2010). Es una aptitud que debe poseer el personal del área de relaciones públicas para poder realizar el proceso de cabildeo con éxito, considerando como herramientas las Actividades de Responsabilidad Social.

\section{METODOLOGÍA DE LA INVESTIGACIÓN}

Esta investigación se limitó a describir la relación entre las Actividades de Responsabilidad Social y el cabildeo o lobbying, con base en las teorías de las relaciones públicas y el pensamiento estratégico.

Así, el proyecto fue integrado en cinco temas fundamentales: las relaciones públicas, la comunicación, el pensamiento estratégico y, por supuesto, las Actividades de Responsabilidad Social y el cabildeo, que fueron los términos a relacionar.
Esto fue pertinente porque tanto las ARS como los procesos de cabildeo están cada vez más presentes en las actividades de las empresas, y hay una relación que es importante aclarar.

Además tuvo relevancia porque anteriormente el contexto político de las empresas era muy autoritario y con poca posibilidad de interacción. Ahora el contexto político permite una interacción más libre, por esta razón resultó importante el cabildeo, que es una forma de relación e influencia sobre los stakeholders.

Este proyecto también fue factible porque el marco teórico pudo aplicarse al caso de la Cooperativa La Cruz Azul, S. C. L.

La investigación se llevó a cabo bajo un análisis cualitativo donde intervinieron tres enfoques: el análisis documental teórico y de caso, la observación no participante y el análisis a profundidad de la entrevista; lo cual fue completamente relevante para el desarrollo del caso Cementera La Cruz Azul. Se utilizó como instrumento de investigación la entrevista a uno de los representantes de la Cooperativa para obtener datos relevantes que permitieron identificar el cabildeo y las ARS en su área de las relaciones públicas, con el fin de poder analizarlos e incluirlos en las páginas de los resultados.

Las entrevistas se realizaron con base en una serie de preguntas previamente elaboradas y considerando la bibliografía que desarrolla este tema. Los días y las horas acordadas se establecieron vía email con la persona a entrevistar. Se hizo uso de anotaciones y de grabadoras como lo permitió el entrevistado. Finalmente, se efectuó una revisión exhaustiva de las respuestas.

Además se realizaron técnicas de investigación documental, como búsqueda de textos científicos, de los cuales ya se hizo mención párrafos arriba, y documentos que la misma empresa elabora, con el objetivo de sustentar lo que se afirma en este proyecto.

La investigación se delimitó a la Industria Cementera en México, para dar cabida al caso de la Cooperativa La Cruz Azul, que es afectada directamente por las Normas Oficiales Mexicanas (NOM). 
La presentación de resultados se observará a lo largo del informe, debido a que la naturaleza de la información permite integrarla en la narrativa.

\section{REPORTE FINAL}

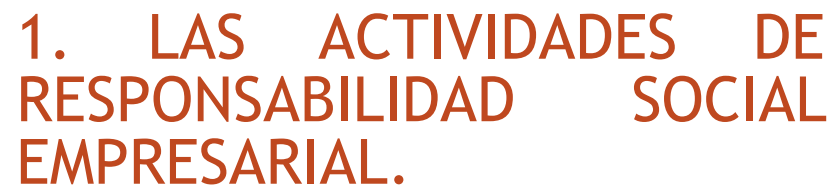

Cada vez hay más empresas que afectan a la sociedad a través de sus actividades. Por ejemplo, una empresa petrolera que contamina los mares y causa la muerte de la fauna marina genera una alteración grave en el ecosistema. Esto, entre otras razones, por la falta de normatividad que pudiera impedir la contaminación.

De ahí que la sociedad haya puesto los ojos en estos actos empresariales, lo cual resulta en desventaja para las organizaciones económicas por los efectos que estos descontentos sociales pudieran causar en sus actividades productivas. En consecuencia, cada vez hay más empresas que generan acciones de responsabilidad social, que aunque no son su obligación legal ni social, las realizan por cumplir con la actividad moral de resarcir los daños causados a la sociedad.

La Responsabilidad social es un término cuya definición se ha perdido en la generalización de la Responsabilidad Social Empresarial (RSE), por esto hay escasez de autores que describan este concepto; en esta investigación se hará uso de una conceptualización propia. En primer lugar, la responsabilidad es una obligación legal o moral a consecuencia de un delito o culpa (RAE; 2015), del mismo modo, la RAE (2015) define el término social como lo referente a la sociedad. Por consiguiente, la responsabilidad social es la obligación moral o legal que se tiene con la sociedad por causarle algún daño.

De este modo la Responsabilidad social es una actividad que se puede desarrollar dentro de las organizaciones económicas. Éste es un tipo de Responsabilidad Social Empresarial que carece de una definición unilateral, por lo que varios autores definen el término dando claridad y veracidad a este proyecto.
Autores como Guerrero (2002) definen la RSE como el compromiso que adquieren las empresas para incorporar en sus actividades diarias planes de trabajo y organización que permitan retribuir a la sociedad lo que toman de ella.

Además de la multitud de definiciones sobre RSE que crean complejidad en el entendimiento del término, existe otro debate que da cabida a la ambigüedad, que es la tendencia a confundir los términos filantropía y RSE.

Por un lado, la filantropía se concibe como un acto de caridad y responsabilidad personal y se considera como parte de la RSE (Carrillo et Al., 2009). Mientras que la RSE implica actos que impacten de forma positiva el desarrollo de la sociedad pero con el propósito de recibir beneficios en el largo plazo.

Continuando con la RSE, el adjetivo de responsabilidad que se le da a las empresas lo adquieren cuando realizan actividades que resarcen social o legalmente el daño causado a los integrantes de la misma empresa o a los grupos de personas fuera de ella.

Por lo anterior, se precisa un análisis más profundo de las ARS. Éstas son actividades que las empresas se ven obligadas legal o moralmente a hacer porque han hecho daño a diversos grupos sociales. De esta forma las empresas evitan que los grupos sociales realicen acciones en contra de ellas, aunque van más allá de esto, buscan legitimarse ante ellos.

Con los grupos sociales se hace referencia a los stakeholders. Como bien mencionan Newman y Mejía (2013), los stakeholders son grupos de interés, "se refiere a cualquier entidad que sea afectada de alguna manera por las operaciones de la empresa o de sus productos o servicios".

Las actividades diarias de la Cooperativa La Cruz Azul afectan a diversos grupos (los stakeholders), como son la sociedad a la que no le llega agua por el uso excesivo de la Cooperativa, los proveedores de energía (antes PEMEX), maquinaria, etc., con que realiza sus actividades, el Gobierno que recibe los impuestos que debe pagar, el Cemefi (Centro Mexicano para la Filantropía) el cual la certifica como ESR, etcétera. Todos los grupos que son afectados por las operaciones y productos que realiza la Cooperativa son stakeholders.

guien o algo conforme a las leyes (RAE, 2015). 
Así pues, las empresas buscan adquirir legitimación ante los stakeholders y algunas formas pueden ser a través del reconocimiento como: Empresa Socialmente Responsable, por el Centro Mexicano para la Filantropía (Cemefi) 2002, 2003, 2004, 2005 y 2006; Premio a la Industria Ética, 2002; Premio Nacional de Ahorro de Energía, Fideicomiso para el Ahorro de Energía Eléctrica (FIDE), Comisión Federal de Electricidad (CFE), 2003, 11 certificados de Industria Limpia; o de 14 certificados ISO 14000 (Newman y Mejía; 2013).

En conjunto, las estrategias de comunicación que hace la empresa deben ser perfectamente planificadas para adquirir legitimidad. Así pues, el término estrategia se refiere a elegir un curso de acción de entre varias opciones; y con respecto a las estrategias empresariales, éstas tienen como propósito ayudar a los administradores a tomar las decisiones sobre qué le conviene a la empresa con base en sus verdaderas prioridades y el alcance de los objetivos de dichas estrategias (Hitt, Ireland, Hoskisson, 2004).

\section{RELACIONES PÚBLICAS DE LAS ORGANIZACIONES}

A las empresas no les basta con comprar insumos, producir, almacenar y vender sus productos, también necesitan generar relaciones sociales con la sociedad en general. De hecho, todo el proceso productivo de una empresa no sería el más eficiente sin las actividades de las relaciones públicas. Por ejemplo, el que se encarga de planear la compra de insumos debe tener buenas relaciones con los proveedores, esto con el fin de que le vendan los productos a un mejor precio, o que éstos sean de excelente calidad; otro ejemplo es la producción, hay que tener buena relación con los empleados del área productiva, para asegurarse de que realizarán su trabajo de la mejor manera, etcétera.

De ahí que las relaciones públicas sean de gran importancia para las empresas. Durante el 1er. Foro Interuniversitario de Investigadores en Relaciones Públicas (2003) se definió a las relaciones públicas como la disciplina que estudia la gestión del sistema de comunicación a través del cual se establecen y mantienen relaciones entre una organización o persona y sus stakeholders.
Es por lo anterior que las actividades de relaciones públicas toman poder dentro de la organización. El poder es un acto que involucra la fuerza o la coerción y es un factor importante dentro de las organizaciones porque quiere decir que quien tiene el poder manda sobre los demás (Hall, 1983). En este caso, las relaciones públicas tienen peso importante en los altos mandos de las organizaciones, por el hecho de que las actividades de relaciones públicas generan un beneficio a la organización al mantener lazos estrechos con otras empresas o personas.

Como consecuencia del poder que tienen las actividades de relaciones públicas en la empresa, es necesario crear un área propia para estas labores, incorporándolas a la estructura organizacional de la empresa y por tanto asignándoles un financiamiento propio para su realización, bajo la dirección y control de un jefe de área o departamento. De cierta manera, el área de las relaciones públicas tendría poder burocrático, es decir, se posicionaría en una estructura jerárquica hasta la cima, con áreas como ventas, compras, producción, etcétera.

Las actividades del área de relaciones públicas se desarrollan secuencialmente y bajo una lógica estratégica. Para Xifra (2003) existen pasos que toda área de relaciones públicas debe desarrollar, y son: planificar, comunicar y evaluar los resultados.

En el primero, hay que identificar los mensajes primarios y secundarios que se transmitirán a los stakeholders, y crear una estrategia específica para que el mensaje enviado sea decodificado de la forma correcta por los stakeholders, lo cual se verá reflejado cuando se tenga la legitimidad deseada.

El segundo paso consiste en poner en marcha la estrategia diseñada en el paso anterior (la planificación), pero siempre verificando en el desarrollo de este proceso que la estrategia sea correcta.

gos transmitidos en forma de mensaje del emisor al receptor. Así pues, los códigos son un conjunto de símbolos y signos que una persona u organización (emisores) quieren transmitir a otra persona u organización (recepto-

tendiendo esos símbolos y signos que le envió el emisor 
El último paso, la evaluación de los resultados, implica corroborar o refutar si se logró influir en los stakeholders, independientemente de hasta qué momento de la influencia se haya querido llegar desde un principio, por ejemplo, cambiar la manera de pensar de los stakeholders o que realicen acciones que desde un principio la empresa planeó para su beneficio propio o simplemente para demostrar el poder de su influencia. El objetivo es influir sin importar hasta qué punto.

Puede ejemplificarse este proceso de relaciones públicas en el caso de La Cruz Azul. La Cooperativa realiza ARS como mantener limpias las áreas verdes y que el agua llegue a todos los hogares de las familias que viven cerca de las fábricas Cruz Azul que fueron usados durante sus actividades productivas, pero como esto no basta, es necesario darlas a conocer, por eso publicitan dichas ARS en anuncios comerciales, adquieren distintivos de ESR, etc. La publicidad de dichas ARS debe ser planeada y puesta en marcha estratégicamente, para finalmente comparar los resultados obtenidos y los planeados, de ello dependerá que se vuelva a seguir la estrategia.

De esta forma resulta importante que quien desarrolle el proceso de las relaciones públicas posea pensamiento estratégico, con el fin de que la estrategia que se diseñe sea la adecuada y no un gasto innecesario para la empresa o disminuya la credibilidad ante los stakeholders.

\section{ESTRATEGIAS DE COMUNICA- CIÓN EN EL ÁREA DE RELACIONES PÚBLICAS}

La empresa está en constante comunicación, tiene la necesidad de hacerse oír y dar a conocer sus productos, servicios, acciones sociales, desarrollo económico, etc., a los stakeholders. Así pues, la comunicación es una acción grupal influenciada por el contexto en el que se desarrolla y está conformada por varias partes que son: el emisor, el receptor, el código, el mensaje, el referente, las barreras, el canal y la interpretación (Ministerio de Fomento de España, 2008).
En el caso de la Cooperativa La Cruz Azul, se pueden distinguir las partes de la acción comunicativa, por ejemplo, es claro ver en la página de internet todas las ARS que realiza la organización, como son la creación de escuelas, pavimentación de las calles donde se encuentran instaladas sus fábricas, instalación de sistema de agua para que llegue a todas las casas, creación de jardines, cines, bibliotecas, etc., para la población de las localidades donde están sus instalaciones; prácticamente todos son miembros de la organización. En este caso, el emisor es La Cruz Azul, el receptor es la sociedad que es afectada por las acciones de la Cooperativa, el gobierno que genera las políticas públicas que pueden afectar de forma negativa o positiva a $\mathrm{La}$ Cruz Azul, es decir, todos los que puedan tener acceso a la página de internet donde se transmite el mensaje.

Así pues, el mensaje sería que la organización se interesa por el bienestar social y prueba de ello es que están realizando ARS para mejorar la calidad de vida de sus miembros, puesto que alrededor de las zonas no hay nada. Este mensaje debe ser claro y conciso, y ser transmitido a través de una serie de símbolos y signos compartidos entre los públicos y La Cruz Azul. El mensaje es afectado por un referente, es decir, por el contexto social, económico y político en el que se envía, y por las barreras que impiden que se entienda.

Las organizaciones económicas realizan actos de comunicación, entre otras cosas, para adquirir legitimidad por parte de los stakeholders, por ello la importancia de planear las estrategias comunicativas. Para este proyecto, es indispensable dirigir la atención del lector hacia el logro de la creación de legitimidad frente a los stakeholders y no a los públicos en general que tienen acceso a la información que la empresa está comunicando. La teoría distingue estos dos términos, ya que los públicos tienen acceso a la información que la empresa comunica, como ya se dijo, pero a la empresa sólo le interesa legitimarse ante los stakeholders, ya que son los grupos sociales afectados o que afectan de forma directa o indirecta a la empresa; si no hubiese influencia alguna, esos grupos sociales le resultan indiferentes a la organización. 
Las estrategias de comunicación de una empresa son elaboradas en el área de las relaciones públicas, entre las actividades que realizan estas áreas, se encuentra crear una buena imagen de la empresa debido a la importancia que se le da a la primera visión que tienen los stakeholders de la misma. También implica tener una buena relación con los medios de comunicación, así como la creación de informes y presentaciones dirigidos a los stakeholders, en fin, todo lo necesario para transmitir un mensaje que beneficiará a la empresa a través de la influencia en la forma de pensar de éstos.

Esto trae consigo el tema de la actualidad, en el que se cuestiona si en verdad las empresas realizan ARS o sólo se quedan en el discurso. Pues independientemente de si lo hacen o no (porque no viene a importar en este tema), la empresa no debe dejar cabida a la duda de que sí las hace y eso se logra a través de eficientes estrategias de comunicación de sus ARS. La forma de comprobar que los stakeholders creyeron en la realización y efectividad de las ARS es cuando la empresa percibe legitimidad ante ellos.

\section{EL CABILDEO EN LAS ORGANIZACIONES ECONÓMICAS} 4.1. EL CABILDEO.

En el área de relaciones públicas de las organizaciones económicas se generan estrategias de comunicación, por ser una actividad importante para la difusión de información. Igualmente resulta importante desarrollar la actividad de cabildeo o lobbying en esta área.

Para comparar la definición citada en este marco teórico se recurre a Graham Wooton (citado en Galaviz, 2006) quien define el lobbying como "personas actuando para influir en las decisiones gubernamentales". "En español el término que se utiliza para esta actividad es 'cabildear' que significa gestionar con actividad y maña para ganar voluntades en un cuerpo colegiado o corporación" (RAE, citado en Galaviz, 2006).

Hay dos formas para que una empresa realice actividades de cabildeo: la interna y la externa.
La primera es ejercida directamente por la organización a través de sus empleados; y la segunda por profesionales del lobbying que son contratados por la empresa para realizar las actividades de cabildeo (Xifra, 1998). Este proyecto sólo considera la investigación y estudio de las actividades de cabildeo internas a la empresa, que forman parte del área de las relaciones públicas, es decir, donde habrá un personal capacitado que esté inmerso en los procesos de la empresa, con mayor acceso a la información, mayor comunicación con los miembros de la empresa, etc., lo cual representa una ventaja que si se contrataran a cabilderos, por el costo que implica su contratación.

El cabildeo podría confundirse con simples actividades de comunicación, ya que su proceso de acción es muy parecido. Mientras que las actividades relacionadas con las estrategias de comunicación implican la planeación, creación y aplicación de las estrategias con el fin de sólo comunicar y adquirir legitimidad, el cabildeo es una actividad más detallada, en este caso se requieren de estrategias de comunicación persuasiva y argumentativa para lograr influir en las decisiones de los stakeholders.

En sí, la finalidad del cabildeo es conseguir que la decisión de los stakeholders se ajuste a los intereses a los cuales sirve la estrategia de comunicación (Xifra, 1998), buscando influir en el andamiaje institucional en el contexto social, económico y político que afecta a la empresa.

\subsection{MARCO INSTITUCIONAL.}

Las empresas realizan sus actividades bajo el cumplimiento de un conjunto de instituciones creadas por un gobierno para controlar dichas actividades empresariales, entonces se dice que se está regulando a la organización económica. La regulación es crear restricciones a las alternativas de los agentes económicos (en este caso las empresas), a través de un mandato legal y formal, siempre y cuando la sociedad considere que debería ser regulada (Amaya, 1997).

Así pues, la regulación consiste en crear instituciones que sistematicen el comportamiento de los individuos y empresas. 
"Las instituciones son las reglas del juego en una sociedad o, más formalmente, son las limitaciones ideadas por el hombre que dan forma a la interacción humana" (North, 1990).

Las instituciones son una parte fundamental para la convivencia de la sociedad, "establecen los incentivos y las reglas que guían la percepción, las preferencias y las decisiones de los individuos y organizaciones" (Oriol, 2007).

El cabildeo consiste en influir en el diseño y proceso de aprobación y puesta en marcha de las instituciones (los tratados internacionales, la constitución, leyes federales, leyes ordinarias, decretos de ley, reglamentos y normas), a través de la influencia en los stakeholders mediante las estrategias de comunicación de las empresas.

"La principal característica de las instituciones es que se ocupa de la ley y del papel central de la ley en la actividad gubernamental, así pues, la ley constituye tanto la estructura del sector público mismo como la herramienta fundamental del gobierno para influir en el comportamiento de los ciudadanos" (Peters, 2003). Por ello la influencia en el gobierno logra influencia en la sociedad.

\subsection{PROCESO DE CABILDEO O LOBBYING.}

Como ya se expuso previamente, los procesos de cabildeo son diferentes, dependen del contexto social, político y económico en el que se desenvuelve la empresa. Por ello, cada autor que se refiere a este tema, incorpora más o menos pasos en el proceso que otros y se enfoca en uno, mientras los demás hacen hincapié en otros.

Sin embargo, los diferentes procesos de cabildeo tienen pasos en común, por ello en este proyecto se consideran los pasos esenciales donde cada empresa los adapta a sus situaciones contextuales, que son:

a)Determinar el problema y dar soluciones. Al principio, la empresa se dará cuenta que una parte del andamiaje institucional le afecta, lo que la impulsará a tomar la decisión de cabildear. Dentro del análisis también se deben determinar quiénes son los stakeholders, para ello se podría utilizar un mapeo de poder para identificarlos con mayor facilidad.
También se debe definir el contexto en que se desarrolla el problema, y/o la parte del andamiaje institucional que se quiere cambiar, así, con base en ello diseñar posibles soluciones. La solución tiene que influir en alguna de las partes del andamiaje institucional, para que esta vez afecten a la empresa positivamente o neutralicen su negatividad anterior.

b)Diseñar la estrategia. Una vez que se conoce el problema institucional que se quiere solucionar y se tienen varias alternativas de solución, es necesario elegir sólo una. Ésta tiene que ser la que genere mayores beneficios a los intereses de la empresa. Una herramienta para seleccionar la mejor alternativa puede ser idear la situación a dónde se quiere llegar y los posibles inconvenientes.

Diseñar la estrategia implica programar el momento adecuado de la intervención para implementar la estrategia comunicativa sobre los stakeholders, además habrá que diseñar formas de hacerlo y la manera de controlar el proceso.

c)Ejecución y control de la estrategia. Una vez establecida la estrategia y acondicionado todo para aplicarla, hay que ponerla en marcha para después vigilar que se esté cumpliendo el objetivo antes establecido.

d)Evaluación de los resultados. Este paso consiste en comparar los resultados reales generados por el proceso de cabildeo y el objetivo establecido durante la etapa dos.

Dicho objetivo debe lograr una influencia en el gobierno para que cambie la institución formal que afecta a la empresa, por otra que anule el daño o la beneficie.

mapeo de poder es un ejercicio de análisis que (Soriano y Ortega, 2004). 
Ejecución y control de

Determinar al problema

y dar soluciones

Diseñar la estrategia

Área de Relaciones

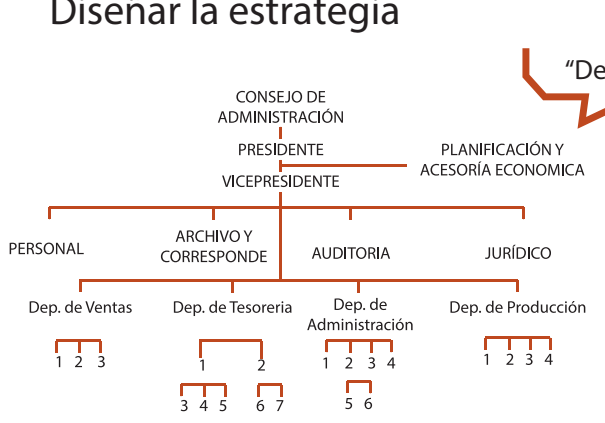

Evaluación de los resultados

rrollo de cabildeo"

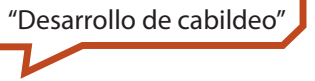

CONVENCERE INFLUIR $\longrightarrow$ la estrategia.

LEGITIMACIÓN

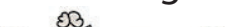

(
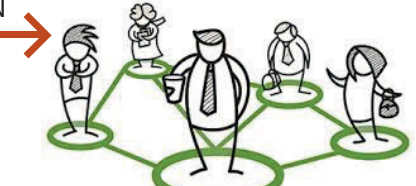

ERARQUIA DE LAS LEYES

CONSTITUCIÓN POLITICA A.INTERNACIONALES

LEYES FEDERALES

REGLAMENTOS FEDERALES NORMAS OFICIALES MEXICANAS

LEYES ESTATALES REGLAMENTOS ESTATALES DECRETOS ESTATALES

STADO

5.CASO: COOPERATIVA LA CRUZ AZUL, S. C. L.

5.1. LA COOPERATIVA LA CRUZ AZUL.

La Cruz Azul es la cooperativa más grande del país dedicada a la fabricación de cemento, creada en el Estado de Hidalgo por el inglés Henry Gibbon, quien después se asoció con otro inglés llamado Joseph Watson. Fue en 1906 cuando los fundadores de la Cooperativa llevaron a la quiebra a la organización. Para 1910 la Cooperativa fue salvada por el socio Fernando Pimentel y Fagoaga, y desde entonces La Cruz azul ha crecido año con año (La Cruz Azul, 2015).

\subsubsection{ACTIVIDADES DE RESPONSABILIDAD SOCIAL DE LA COOPERATIVA LA CRUZ AZUL.}

La Cooperativa La Cruz Azul publicita un gran número de ARS en su página de internet, en sus informes anuales, en la televisión, la radio, reconocimientos y distintivos obtenidos, entre otros. Las ARS que presume de realizar la Cementera son: brindar servicios de educación y salud a las comunidades aledañas a las fábricas de la cooperativa a través del Club Deportivo Social y Cultural Cruz Azul, Fundación Cruz Azul ProComunidades, Centro Clínico Pedagógico Integral Cruz Azul, Centro de Administración de Servicios de Salud, Programa Desarrollo Humano Integral Cruz Azul DHICA, Asociación de Socios Jubilados y el Club de Aficionados al Equipo Cruz Azul.

Aunado a lo anterior, podría considerarse como ARS que La Cruz Azul diseña sus plantas cementeras para "el consumo eficiente de energía tanto eléctrica como térmica y todos los esfuerzos están encaminados al ahorro de agua y a evitar la contaminación por polvos.

Ejemplo claro es la cuarta fábrica de cemento de La Cruz Azul, ubicada en Palmar de Bravo, en el Estado de Puebla, que cuenta con sistemas de muy avanzada tecnología anticontaminante ya que el $12 \%$ de la inversión total fue destinada a la adquisición de equipo especializado para este fin" (Cooperativa La Cruz Azul, 2015).

Otro ejemplo es la planta Lagunas, Oaxaca, que invirtió en un sistema de agua para que se reutilizara en los procesos productivos de la Cooperativa y una vez expulsada de la planta saliera como agua limpia para el consumo de las casas aledañas, según el ingeniero Domínguez.

También actualiza constantemente sus procesos productivos, supervisa y da mantenimiento a la infraestructura operativa, lo cual según la Cooperativa garantiza el cumplimiento de la legislación ambiental y permite disminuir los riesgos de trabajo, cumpliendo así con algunas normas de seguridad e higiene.

En suma, la Cooperativa La Cruz Azul realiza muchas ARS, tanto internas como externas a la organización. Sin embargo, esto no le basta, por eso se encarga de comunicarlas a través de la obtención de certificados y distintivos, los informes anuales que realiza la empresa, páginas de internet, televisión, radio y prensa. Estas estrategias de las relaciones públicas de la Cementera son una forma de adquirir legitimidad ante sus stakeholders e influir también sobre ellos. 


\subsubsection{LAS NORMAS OFICIALES MEXICANAS (NOM).}

Las Normas Oficiales Mexicanas (NOM) son las instituciones más específicas del Marco Normativo en México. Según la Secretaría de Economía (2015), las NOM “son regulaciones técnicas de carácter obligatorio. Regulan los productos, procesos o servicios, cuando éstos puedan constituir un riesgo para las personas, animales y vegetales así como el medio ambiente en general, entre otros".

En el caso específico de la Cementera, ésta cuenta con una serie de NOM que la regulan. "La normatividad para los cementos en México, está a cargo del Organismo Nacional de Normalización y Certificación de la Construcción y Edificación (ONNCCE). Para emitir las normas correspondientes a los cementos hidráulicos, el ONNCCE formó un Comité donde participan, entre otros, los Técnicos responsables de la calidad de cada Empresa Cementera en el país, Institutos y Universidades, en el cual tienen una participación activa los Técnicos de La Cruz Azul” (Cooperativa La Cruz Azul, 2015).

Desde esta perspectiva, la Cooperativa realiza ARS y las incorpora a sus estrategias de relaciones públicas para conseguir legitimidad e influir en los stakeholders, al mismo tiempo que puede influir en las NOM. Se dice que La Cruz Azul está haciendo procesos de cabildeo.

\subsection{PROCESO DE CABILDEO EN LA COOPERATIVA LA CRUZ AZUL.}

En un análisis más profundo del proceso de cabildeo que se le está atribuyendo a la Cementera, se desarrollan cada uno de los pasos de que consta el proceso:

\section{Determinar el problema y dar soluciones.}

En sí, no hay un problema latente en el que las NOM afecten a la Cooperativa de forma negativa o que a la organización le interese cambiar. Sin embargo, las ARS son una forma de mantener la legitimidad ante los stakeholders y con ello que las NOM no dañen a la organización en un futuro.

En el caso de la fábrica en Oaxaca, la Cooperativa puede influir en las decisiones gubernamentales, ya que la organización contribuye a la realización de eventos que el gobierno realiza en el pueblo de Lagunas, por ejemplo en la visita de Rosario Robles, Secretaria de Desarrollo Agrario, Territorial y Urbano de México (SEDATU) el 29 de octubre del presente año, para la inauguración de una obra a la cual La Cruz Azul había donado materiales para su construcción, según el Ing. Domínguez.

\section{Diseñar la estrategia.}

Las estrategias en este caso están a cargo del área de relaciones públicas que comunica eficientemente las ARS de La Cruz Azul, para poder legitimarse e influir en los stakeholders. Por ejemplo, la mayor parte de sus informes anuales están dirigidos a describir las acciones que La Cruz Azul hace en favor de la sociedad, adquieren una gran cantidad de distintivos que garantiza a la sociedad la efectividad de la ARS de la Cooperativa (independientemente de si dichas actividades se realizan o no), pagan por un amplio número de reproducciones de anuncios comerciales en televisión y radio, gastan en los sistemas de agua potable, en la limpieza de áreas verdes y creación de escuelas y centros recreativos en las localidades donde se encuentran instaladas sus fábricas.

\section{Ejecución y control de la estrategia.}

Este paso se ve reflejado en los informes anuales, las páginas de internet, radio y televisión. Por ejemplo, en la planta de Oaxaca hay un gran número de anuncios que informan de las ARS que realiza la Cooperativa, las paradas de autobús tienen su logo, hay espacios reservados para comunicarle a la colonia de sus actividades, los monumentos están sellados con la marca Cruz Azul, entre otros.

\section{Evaluación de los resultados.}

El objetivo aquí es influir en las NOM en favor de los intereses de la Cooperativa La Cruz Azul. Así pues, el resultado hasta el momento ha sido positivo, ya que la Cooperativa no ha presentado nuevos problemas por el marco institucional.

Las contribuciones que hace la Cooperativa para los actos gubernamentales tienden a favorecerla, como comentó el Ing. 
Domínguez, le dan a La Cruz Azul la oportunidad de intervenir en las decisiones políticas. Esto de alguna forma afecta el marco institucional donde se encuentra inmersa.

En la gráfica 3 se muestra el proceso simplificado de cabildeo que realiza la Cooperativa utilizando sus ARS.

cabildeo: Caso La Cruz Azul.

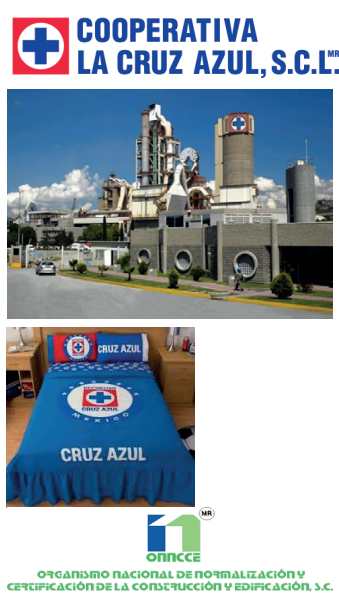

Determinar el problema y

dar soluciones.

Diseñar la estrategia.

Certificaciones y reconocimiento

El ISO 9,000.

Industria Limpia.

Excelencia Ambiental.

Empresa Socialmente Responsable

Corona de la Calidad.

2 Ejecución y control de la estrategia

3 Evaluación de los resultados.

Fuente. Elaboración propia.

\section{CONCLUSIONES}

Al principio de este proyecto se propuso describir la relación existente entre las ARS y el cabildeo a través de la teoría de las relaciones públicas y el pensamiento estratégico, considerando como caso de estudio la cementera La Cruz Azul.

Para la realización de la relación descriptiva se hizo un análisis teórico de estudios previos, de información periodística, informes de la Cooperativa La Cruz Azul y una entrevista a un miembro de la Cooperativa en la fábrica de Oaxaca.

Como resultado se obtuvo que las actividades son utilizadas como herramienta en el proceso comunicativo del cabildeo realizado por el área de relaciones públicas, con el fin de intervenir en la normatividad que afecta a la empresa. Esto se comprueba, ya que las ARS atraen voluntades a favor, en este caso, las de los stakeholders que son los grupos de interés para la empresa. A través de esta influencia, se puede finalmente intervenir en los procesos normativos que afectan a la organización, ya que como sociedad las leyes y regulaciones se hacen conforme al bienestar de la sociedad, entre ésta los stakeholders.

También se concluye que la Cooperativa La Cruz Azul realiza procesos de cabildeo utilizando sus ARS como herramientas comunicativas. Adquiere cierto reconocimiento para influir en la normatividad que le afecta, como el caso de las actividades gubernamentales que se realizan en la Cuidad de Lagunas, Oaxaca y donde La Cruz Azul interviene como organizador, tomando decisiones en esos eventos y normas que le afectan.

De esta manera, resalta la importancia de introducir este nuevo término de cabildeo en el ámbito administrativo, ya que su utilización en teorías administrativas no es bien conocido. Así pues, este proyecto abre la brecha de análisis para futuras investigaciones. 


\section{BIBLIOGRAFÍA}

1. Amaya, M. L. (1997). Oportunidades y obstáculos para la autonomía administrativa en México: El caso de la comisión federal de competencia. México: Centro de Investigación y Docencias Económicas.

2.CARRILLO, Patricia (2009). Diagnóstico sobre filantropía corporativa en México. México: Alternativas y capacidades.

Disponible en: http://www.filantropia.itam.mx/docs/

DiagnosticoFilCorp.pdf [Consultado el: 05 de junio de 2015].

3.CEMEFI (2015) Distintivo ERS 2015. Disponible en: http://

www.cemefi.org/esr/pdf/2015/

Lista\%20empresas\%20ESR\%202015\%20OK\%202110.pdf

4.CENTRO NACIONAL DE METROLOGÍA (2015). Marco

normativo. Disponible en: http://www.cenam.mx/inf_normativa/ [consultado el: 19 de julio de 2015].

5.Comunicación y relaciones institucionales (nivel 1).

Disponible en:

http://www.apalmeria.com/images/stories/file/Empleo/

TecnicoFacturacion_20150430/ManualesFormativos/

Comunicaci\%C3\%B3nYRelacionesInstitucionalesNivel1.pdf

[Consultado el: 03 de junio de 2015], Ministerio de fomento,

España, 2015.

6.COOPERATIVA LA CRUZ AZUL (2015). Comunicación

sobre el Progreso. The Global Compact. Disponible en: http://

cruzazul.com.mx/2008/pdf/CruzAzulCOP_2010.pdf

7.COOPERATIVA LA CRUZ AZUL (2015). Planta Oaxaca.

Disponible en: http://www.cruzazul.com.mx/2008/producto/

plantaOaxaca.aspx [Consultado el 3 de noviembre de 2015]

8.COOPERATIVAS DE LA AMÉRICAS: Región de la

alianza Cooperativa Internacional (2015). Principios y valores

cooperativos. Disponible en: http://www.aciamericas.coop/

Principios-y-Valores-Cooperativos-4456 [Consultado el 3 de noviembre de 2015]

9.Corona, Miguel (2012) "Desarrollo de pensamiento estratégico en la escuela de negocios". Contaduría y administración. Vol.

57(1): 103-122, https://doi.org/10.22201/fca.24488410e.2012.197

10.CORREA, María; Flynn, Sharon y Ami1, Alon (2004).

Responsabilidad social corporativa en América Latina: una visión empresarial. Santiago: CEPAL, Publicaciónı de las Naciones

Unidas.

11.GALAVIZ, E. (2006). El cabildeo legislativo y su regulación.

México: UNAM.

12.GERSTEIN, M. S. (1996). Pensamiento estratégico.

Encuentro en la tecnología. Estrategias y cambios en la era de

la información. México: Addison-Wesley Iberoamericana. Pp.

39-59.

13.HALL, Richard (1983) Organizaciones, estructuras y procesos

( $3^{\text {a }}$ ed.). México: Ed. Prentice/Hall international.
14.Hitt, M., Ireland, R., y Hoskisson, R. (2004). Administración estratégica: competitividad, y conceptos de globalización.

México: Thomson.

15.HULA, Kevin (2002). Cabildeo/Lobbying. México: Limusa.

16.Ministerio de Fomento de España. Comunicación y relaciones institucionales (nivel 1). España.

17.NEWMAN, Bruno y MEJÍA, Martha (2013). Responsabilidad social total. Comunicación estratégica para la sustentabilidad.

México: Fondo de cultura económica.

18.NORTH, Douglass (1990). Instituciones, cambio institucional y desempeño económico. México: Fondo de Cultura Económica. 19.ORIOL, P., J. (2007). "Revisión crítica de los aportes del institucionalismo a la teoría y la práctica del desarrollo". Revista de economía institucional. Vol. 9(16): 121-148.

20.ORTEGA, Miguel y SORIANO, María, Manual de cabildeo.

Disponible en: http://www.portalsida.org/repos/

Manual\%20de\%20Cabildeo.pdf

21.Real Academia Española (2016). Disponible en: http://

www.rae.es/

22.Schwartz, M. y Carroll, A. (2003). "Corporate Social

Responsibility: A three-Domain Approach”. Business Ethics

Quarterly. Vol. 13(4): 503-530,

https://doi.org/10.5840/beq200313435

23.SECRETARÍA DE ECONOMÍA (2015). Catálogo

Mexicano de Normas. Disponible en: http://www.economia.

gob.mx/comunidad-negocios/competitividad-normatividad/

normalizacion/catalogo-mexicano-de-normas [consultado el 20

de julio de 2015].

24.XIFRA, Jordi (1998). Lobbying: Cómo influir eficazmente en las decisiones de las instituciones públicas. Barcelona, España:

Gestión 2000.

25.XIFRA, Jordi (2003). Planificación estratégica de las

relaciones públicas. Barcelona, España: Paidós Ibérica.

Copyright (c) 2017 Alma Isela Rodríguez Hernández

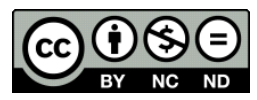

Este texto está protegido por una licencia Creative Commons 4.0.

Usted es libre para Compartir (copiar y redistribuir el material en cualquier medio o formato), siempre que cumpla las condiciones de:

Atribución: Usted debe dar crédito a la obra original de manera adecuada, proporcionar un enlace a la licencia, e indicar si se han realizado cambios. Puede hacerlo en cualquier forma razonable, pero no de forma tal que sugiera que tiene el apoyo del licenciante 0 lo recibe por el uso que hace de la obra.

No Comercial: Usted no puede hacer uso de la obra con propósitos comerciales

Sin Derivadas: Si remezcla, transforma o crea a partir de la obra, no podrá distribuir la obra modificada

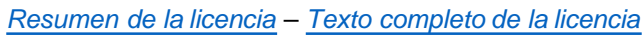

